\title{
Effective collecting area of lobster eye optics and optimal value of effective angle
}

\author{
V. Tichý ${ }^{1}$ (D) M. Barbera ${ }^{2,3} \cdot$ R. Hudec ${ }^{4,5} \cdot$ R. Willingale ${ }^{1}$
}

Received: 29 August 2018 / Accepted: 31 January 2019 / Published online: 14 February 2019

(C) The Author(s) 2019

\begin{abstract}
Effective collecting area represents one of principal parameters of optical systems. The common requirement is to obtain as large effective collecting area as it is possible. The paper presents an analytical method of calculating effective collecting length and its maximization for lobster eye optics. The results are applicable for a Schmidt as well as for an Angel lobster eye geometry used in an astronomical telescope where the source is at infinity such that the incoming rays are parallel. The dependence of effective collecting area vs. geometrical parameters is presented in a form of a simple compact equation. We show that the optimal ratio between mirrors depth and distance (effective angle) does not depend on other geometrical parameters and it is determined only by reflectivity function, i.e. by mirrors (or their coating) material and photon energy. The results can be also used for approximate but fast estimation of performance and for finding the initial point for consequent optimization by ray-tracing simulations.
\end{abstract}

Keywords Lobster eye · Multi-foil optics · Reflective optics · Grazing incidence optics $\cdot$ X-ray optics

V. Tichý

vladimir-tichy@email.cz

1 Department of Physics and Astronomy, University of Leicester, University road,

LE1 7RH Leicester, UK

2 Dipartimento di Fisica e Chimica, Università degli Studi di Palermo, Via Archirafi 36, 90123 Palermo, Italy

3 Istituto Nazionale di Astrofisica, Osservatorio Astronomico di Palermo G.S. Vaiana, Piazza del Parlamento 1, 90134 Palermo, Italy

4 Faculty of Electrical Engineering, Czech Technical University in Prague, Technická 2, 16627 Praha 6, Czech Republic

5 Engelhardt Astronomical Observatory, Kazan Federal University, Kremlyovskaya street 18, 420008 Kazan, Russian Federation 


\section{Introduction}

Lobster eye (abbreviation LE) X-ray optics was proposed a long time ago in two basic concepts: Schmidt[12] and Angel[2].

Schmidt lobster eye[12] can be one-dimensional or two-dimensional. The basic one-dimensional Schmidt lobster eye stack is schematically drawn on Fig. 1. In a real case, grazing angles are much smaller and mirrors are closer one to other.

The system is composed of flat rectangular mirrors (plates). These mirrors form an uniform pattern around of a virtual cylinder of centre $\mathbf{C}$ and radius $r$. Point $\mathbf{F}$ represents the focus of the system. Focal length of the optics equals $f=r / 2$ if it is composed of mirrors of negligible thickness. A two-dimensional Schmidt system is composed of two stacks of different radii $r_{1}, r_{2}$ perpendicularly arranged. Angel lobster eye optics[2] is composed of square pores. It can be viewed as a special case of the Schmidt system, where both stacks lay in the same position and they have the same radii $r=r_{1}=r_{2}$. In such case, two stacks of mirrors form square pores.

The LE optics was used and it is proposed to be used in plenty of astronomical instruments, e.g. $[1,3,5-8,11,18]$. It is being used also in other applications, e.g. neutron imaging [21].

For numerical simulations of lobster eye optics, a general ray-tracing approach is possible, see e.g. [13] or its simplified procedures [20, 21]. Analytic models represents another type of computation. They are useful for approximate but fast estimation of performance. Analytic models are also useful for finding the initial point for consequent optimization by ray-tracing simulations. In addition, analytical models give an understanding of how the results depend of initial parameters. Some analytical equations for lobster system were presented in sources [2, 10, 12] but they do not include all parameters, e.g. zero mirror thickness is supposed. Paper [4] presents a comprehensive analytical analysis but the source is taken to be at a finite distance and the source is on the concave side of LE optics. This configuration is relevant for e.g. a microscope and the configuration is different for telescopes. For application on telescopes, the source must be placed at infinite distance on the convex side of LE optics. Paper [17] presents analysis of focussing efficiency depending on X-ray wave length but the final results are based on ray-tracing simulations of

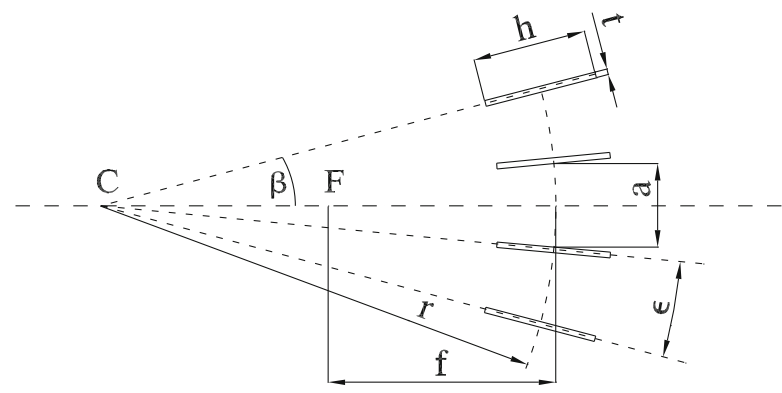

Fig. 1 Lobster eye geometrical parameters 
specific examples. Papers $[14,15]$ present analytical calculations for X-ray optics composed of mirror shells, particularly Wolter optics.

This paper follows previous work of some authors [19] in which equation for calculation of effective collecting area (length) including all geometric parameters are presented however the method for taking the mirror reflectivity into account is only covered in outline. In this paper, an equation for calculation of effective collecting area/length is modified into a compact form. The equation contains all geometrical parameters and requires a model of mirror reflectivity. The system is supposed in a telescope configuration, i.e. incoming rays are parallel and an image is acquired in the focus of the optics. The equation is applied using three models of reflectivity incorporating a wide selection of materials. For these models, the maximum possible effective collecting area/length is found.

\section{Decomposition of dimensions and effective length}

The simplified drawing of Schmidt lobster eye is seen on Fig. 2. There are two stacks of mirrors: vertical stack of width $b_{1}$ and height $a_{1}$ and horizontal stack of width $a_{2}$ and height $b_{2}$.

The mirrors of the first stack (the right one on Fig. 2) act on reflection on its full height $b_{1}$. However, because some mirrors fully or partially lays in shades of other mirrors, effectively, the collection size of the stack in horizontal dimension is limited. This reduced size we call effective collecting length $L_{1}$. The effective collecting area (as it is usually defined) of first stack (if it is alone) equals $L_{1} b_{1}$.

By analogy, the mirrors of the second stack act on reflection on its full width $b_{2}$. The collection length in vertical dimension $a_{2}$ is reduced to a length $L_{2}$. The effective collecting area (as it is usually defined) of the second stack (if it is alone) equals $L_{2} b_{2}$.

In the entire system of both stacks as it drawn on Fig. 2, it is supposed that outer dimensions of stacks are similar, i.e. $a_{1} \approx b_{2}$ and $a_{2} \approx b_{1}$, while $L_{1}$ is significantly less than $a_{1}$ and $L_{2}$ is significantly less than $a_{2}$. Therefore, the vertical size of first stack $a_{1}$ does not affect the collecting size of second stack in vertical dimension or vice versa and the effective collecting area of entire system composed of both stacks equals $L_{1} L_{2}$.

If the system is of Angel type, composed of square pores, it is possible to approximately model it as a system of two identical orthogonal stack of mirrors laying at the

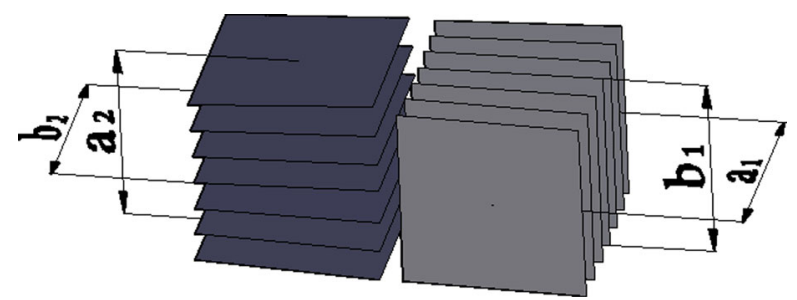

Fig. 2 Dimension decomposition 
same position. If $L$ is effective collecting length of one of such stack, the effective collecting area of entire system equals $L^{2}$.

\section{Effective collecting length - general equation}

The derivation of the equation for the effective collecting length is made in a similar way as it is done in the paper [12]. Moreover, we added reflectivity as it is outlined in the paper [19]. As in papers [4, 12, 17, 19, 20], the problem of one-dimensional lobster eye stack is analysed in cross-section and the geometry becomes just twodimensional.

The geometry of the stack of mirrors is described by parameters (Fig. 1): radius of the system $r$, size of space between centres of surfaces of adjacent mirrors (pore width) $a$, plate thickness (or width of wall of pore) $t$, plate (or pore) depth $h$ and number of plates (number of pores in one dimension) $N$. Variable $\beta$ represents the angular position of a mirror. The incoming rays are considered to be parallel with line $\mathbf{C F}$ and therefore $\beta=\theta$, where $\theta$ is a grazing angle.

The effective angle $\zeta$ of the lobster eye system, we define as the maximal possible grazing angle when the mirror does not lay in a shade of the adjacent mirror. see Fig. 3a [10, 12, 19]. At this angle, the mirror is fully illuminated and only one ray can theoretically go through the optics directly between the illuminated mirror and the adjacent mirror. Because radius of the system is usually much bigger than the mirror dept $h$, for the purpose of definition, adjacent mirrors are considered to be approximately parallel. Evidently, $\zeta \approx \arctan (a / h) \approx a / h$.

The limiting angle $v$ of the lobster eye system, we define as the maximal grazing angle when a single reflection is theoretically possible for a ray, see Fig. $3 b$ [10, 12, 19]. Evidently, $v \approx \arctan (2 a / h) \approx 2 a / h=2 \zeta$.

If a grazing angle $\theta$ is less or equal to the effective angle $\zeta$ then a mirror is fully illuminated. The mirror contributes to the total effective collecting length by the length equal to the orthogonal projection $l_{1}(\theta)$ of its total length to the ray direction, see Fig. 4a.

The equation for the length $l_{1}(\theta)$ equals [12].

$$
l_{1}(\theta)=h \sin \theta \approx h \theta .
$$

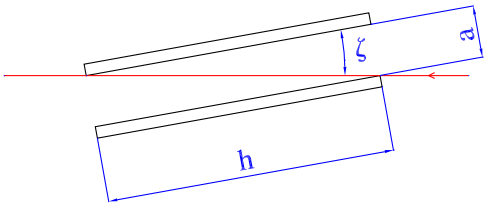

(a) Effective angle

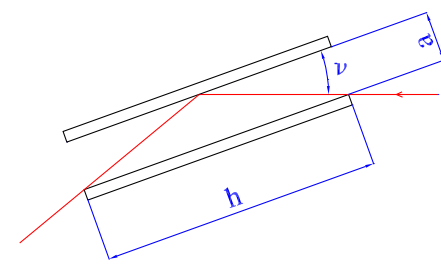

(b) Limiting angle

Fig. 3 Definition of Effective angle $\zeta$ and limiting angle $v$ 


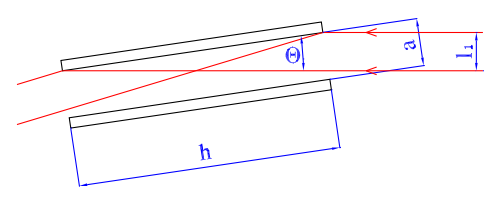

(a) $0 \leq \theta \leq \zeta$

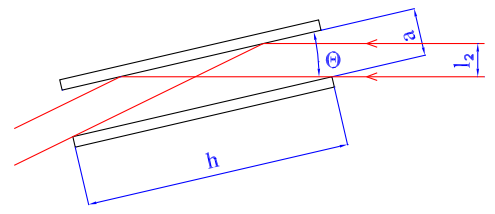

(b) $\zeta<\theta<2 \zeta$

Fig. 4 Effective collecting lengths of individual mirrors

If a grazing angle $\theta$ is greater than the effective angle $\zeta$ but less than the limiting angle $2 \zeta$ then the active area of the mirror is limited by the adjacent mirror, see Fig. 4a. The equation of the orthogonal projection $l_{2}(\theta)$ of the active part to ray direction equals[12]:

$$
l_{2}(\theta)=2 a \cos \theta-h \sin \theta \approx 2 a-h \theta
$$

The total effective collecting length $L$ equals to the sum of effective lengths of all active mirrors. Moreover, the effective collecting length is decreased by reflectivity $\mathcal{R}(\theta)$ of the mirror surface at grazing angle $\theta$, i.e.

$$
L=\sum_{i} l_{1}\left(\theta_{i}\right) \mathcal{R}\left(\theta_{i}\right)+\sum_{j} l_{2}\left(\theta_{j}\right) \mathcal{R}\left(\theta_{j}\right) .
$$

Here, $\theta_{i}$ is a grazing angle of $i$-th mirror. Index $i$ comes through all mirrors fulfilling $0 \leq \theta \leq \zeta$ and index $j$ comes through all mirrors fulfilling $\zeta<\theta<2 \zeta$. Similarly to [12], an approximation of the sums by integrals is used. The source is assumed to be at infinite distance but it may not be on-axis. The calculations are valid also for off-axis source positions if there exist mirrors at all angles between $\pm v$.

$$
L \approx 2\left[\int_{0}^{\zeta} \frac{l_{1}(\zeta)}{\epsilon} \mathcal{R}(\zeta) \mathrm{d} \zeta+\int_{\zeta}^{2 \zeta} \frac{l_{2}(\zeta)}{\epsilon} \mathcal{R}(\zeta) \mathrm{d} \zeta\right]
$$

The value $\epsilon$ denotes the angle pitch of mirrors, i.e.

$$
\epsilon \approx \frac{a+t}{r} \text {. }
$$

The approximation by the integrals is eligible if $\epsilon<<\zeta$, which is usually the case. The factor 2 preceding the square bracket in (4) is there because of the symmetry. It is supposed that mirrors are placed at least in all angle positions between $\pm 2 \zeta$, i.e. their number $N$ fulfils

$$
N>4 \frac{a}{a+t} \frac{r}{h} .
$$

After substitution (1)-(2) to (4), it is obtained

$$
L \approx \frac{2 r}{a+t}\left[\int_{0}^{\zeta} h \theta \mathcal{R}(\theta) \mathrm{d} \theta+\int_{\zeta}^{2 \zeta}(2 a-h \theta) \mathcal{R}(\theta) \mathrm{d} \theta\right],
$$

Integration by parts reads

$$
\int_{p}^{q} \theta \mathcal{R}(\theta) \mathrm{d} \theta=[\theta \overline{\mathcal{R}}(\theta)]_{\theta=p}^{\theta=q}-\int_{p}^{q} \overline{\mathcal{R}}(\theta) \mathrm{d} \theta,
$$


where $\overline{\mathcal{R}}(\theta)$ is an arbitrary first antiderivative of $\mathcal{R}(\theta)$, i.e. $\overline{\mathcal{R}}(\theta)=\int \mathcal{R}(\theta) \mathrm{d} \theta$ and $h=a / \zeta$, Eq. 7 can be modified to a form

$$
\begin{aligned}
L= & \frac{2 r}{a+t}\left\{\frac{a}{\zeta}[\theta \overline{\mathcal{R}}(\theta) \mathrm{d} \theta]_{\theta=0}^{\theta=\zeta}-\frac{a}{\zeta} \int_{0}^{\zeta} \overline{\mathcal{R}}(\theta) \mathrm{d} \theta-\frac{a}{\zeta}[\theta \overline{\mathcal{R}}(\theta)]_{\theta=\zeta}^{\theta=2 \zeta}\right. \\
& \left.+\frac{a}{\zeta} \int_{\zeta}^{2 \zeta} \overline{\mathcal{R}}(\theta) \mathrm{d} \theta+2 a \int_{\zeta}^{2 \zeta} \mathcal{R}(\theta) \mathrm{d} \theta\right\},
\end{aligned}
$$

consequently

$$
L=\frac{2 r a}{(a+t) \zeta}\left[\int_{\zeta}^{2 \zeta} \overline{\mathcal{R}}(\theta) \mathrm{d} \theta-\int_{0}^{\zeta} \overline{\mathcal{R}}(\theta) \mathrm{d} \theta\right]
$$

Equation 10 can be modified to a compact form

$$
L(r, a, t, \zeta)=2 r \frac{a}{a+t} \frac{\widetilde{\mathcal{R}}(2 \zeta)-2 \widetilde{\mathcal{R}}(\zeta)+\widetilde{R}(0)}{\zeta},
$$

where $\widetilde{\mathcal{R}}(\theta):=\iint \mathcal{R}(\theta) \mathrm{d} \theta \mathrm{d} \theta=\int \overline{\mathcal{R}}(\theta) \mathrm{d} \theta$ is an arbitrary second antiderivative of $\mathcal{R}$. The last fraction in (11) we denote as

$$
K(\zeta):=\frac{\widetilde{\mathcal{R}}(2 \zeta)-2 \widetilde{\mathcal{R}}(\zeta)+\widetilde{R}(0)}{\zeta}
$$

Equation 11 allows us to calculate the effective collecting length of a lobster eye of given geometrical parameters and given model of reflectivity.

Equation 11 clearly shows the dependence of the performance with respect to the parameters. First, it is seen that effective collecting length is proportional to the radius of the system. Next, the effective collecting length is proportional to $a /(a+t)$. This term can be interpreted as a ratio between the total size of input aperture and the fraction that is shaded by thickness of mirrors[10, 19]. For a system composed of ideal mirrors of zero thickness, this fraction would equal 1.

The last fraction in (11), i.e. the function $K$ depends on effective angle and reflectivity function only. Because integration of $\mathcal{R}$ is not possible to do in a general way, calculation of $K$ and its maximization is demonstrated in following sections using approximate but reasonable physical models.

Note that if the mirrors are ideal (having $100 \%$ reflectivity), then $\mathcal{R}(\zeta)=1$, $K(\zeta)=\zeta$ and consequently $L=2 r \frac{a}{a+t} \zeta=2 r \frac{a^{2}}{(a+t) h}$. This result is equivalent to equations presented in $[10,12,19]$ where $100 \%$ reflectivity is supposed. For ideal mirrors, larger $\zeta$ leads to larger effective input length and there is no optimal value.

In following sections, it is proven that for real mirrors, there exists an optimal value of $\zeta$, i.e. there exists an optimal ratio between mirror spacing $a$ and depth $h$ when the function $K$ (and consequently the effective collecting length/area) is maximised. 


\section{Single step model}

As the first example, the method is applied for single step model. Here, the reflectivity is approximated by the function

$$
\mathcal{R}(\theta)=\left\{\begin{array}{l}
Q \forall 0 \leq \theta<\kappa \\
0 \quad \forall \theta>\kappa
\end{array}\right.
$$

Here, $\theta$ is the grazing angle and $\kappa$ is a constant known as the critical angle. $0<Q \leq 1$ is a constant, usually $Q=1$. The second antiderivative of the reflectivity defined by (13) equals

$$
\widetilde{\mathcal{R}}(\theta)=\iint \mathcal{R}(\theta) \mathrm{d} \theta \mathrm{d} \theta=Q \begin{cases}-\frac{\theta^{2}}{2}-\kappa \theta+\frac{\theta^{2}}{2} & \forall 0 \leq \theta \leq \kappa \\ 0 & \forall \theta \geq \kappa\end{cases}
$$

The function $K$ is evaluated by substituting (14) to (12). After manipulation we obtain

$$
K(\zeta)=Q\left\{\begin{array}{lr}
\zeta & \forall 0 \leq \zeta \leq \frac{\kappa}{2} \\
-\zeta+2 \kappa-\frac{\kappa^{2}}{2 \zeta} & \forall \frac{\kappa}{2} \leq \zeta \leq \kappa \\
\frac{\kappa^{2}}{2 \zeta} & \forall \kappa \leq \zeta
\end{array}\right.
$$

An example of function $K$ vs. $\zeta$ is shown in Fig. 5, parameters $Q=1$ and $\kappa=$ $100 \mathrm{mrad}$ were chosen.

The final equation for the effective collecting length is obtained substituting (15) into (11) and equals

$$
L(r, a, t, \zeta)= \begin{cases}2 r \frac{a}{a+t} Q \zeta & \forall 0 \leq \zeta \leq \frac{\kappa}{2} \\ 2 r \frac{a}{a+t} Q\left(-\zeta+2 \kappa-\frac{\kappa^{2}}{2 \zeta}\right) & \forall \frac{\kappa}{2} \leq \zeta \leq \kappa \\ 2 r \frac{a}{a+t} Q \frac{\kappa^{2}}{2 \zeta} & \forall \kappa \leq \zeta\end{cases}
$$

The function $K(\zeta)$ has one maximum on $\zeta \in[0, \infty)$, that is

$$
\zeta_{\text {optimal }}=\frac{\sqrt{2}}{2} \kappa \doteq 0.707 \kappa
$$

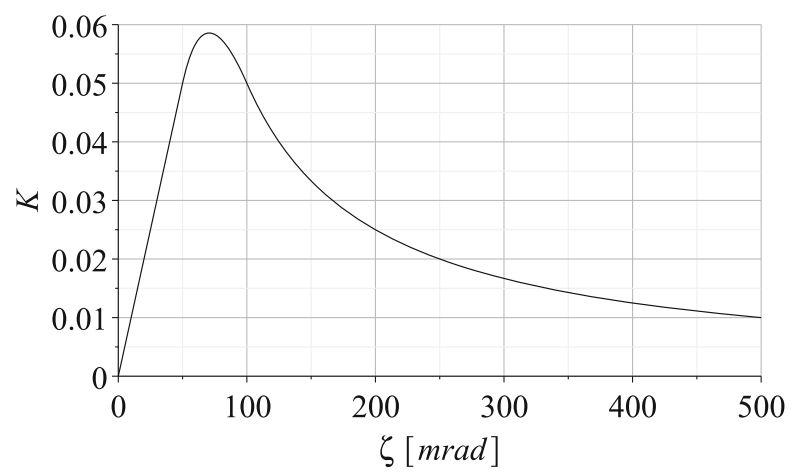

Fig. 5 Example of function $K$ vs. effective angle $\zeta$ for single step model 
If $\zeta=\zeta_{\text {optimal }}$, then the effective collecting length is maximised. This result is presented also in paper [4], where a similar model of reflectivity is supposed for a microscope configuration.

\section{Linear model}

In this section, the reflectivity is approximated by the function

$$
\mathcal{R}(\theta)= \begin{cases}Q\left(1-\frac{\theta}{\kappa}\right) & \forall 0 \leq \theta \leq \kappa \\ 0 & \forall \theta \geq \kappa\end{cases}
$$

Here, $\theta$ is the grazing angle and $\kappa>0$ and $0<Q \leq 1$ are constants. Example of proper linear approximation is shown in Fig. 6a for gold surface and unpolarized $\mathrm{X}$-rays of energy $1 \mathrm{keV}$. Here, $\kappa=84.3 \mathrm{mrad}$ and $Q=1$. Reflectivity data were acquired from [9]. Another example is Iridium at unpolarised X-rays of energy $3 \mathrm{keV}$ of microroughness $1 \mathrm{~nm}$, here $\kappa=32 \mathrm{mrad}$ as shown in Fig. $6 \mathrm{~b}$.

The corresponding equation for $K$ equals

$$
K=Q\left\{\begin{array}{lc}
-\frac{\zeta^{2}}{\kappa}+\zeta & \forall 0 \leq \zeta \leq \frac{\kappa}{2} \\
\frac{\zeta^{2}}{3 \kappa}-\zeta-\frac{\kappa^{2}}{6 \zeta}+\kappa & \forall \frac{\kappa}{2} \leq \zeta \leq \kappa \\
\frac{\kappa^{2}}{6 \zeta} & \forall \kappa \leq \zeta
\end{array}\right.
$$

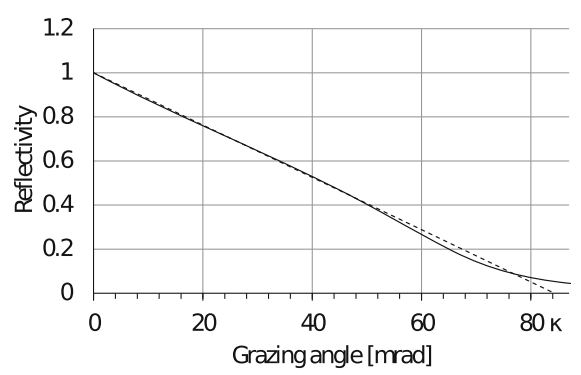

(a) Gold at $1 \mathrm{keV}$

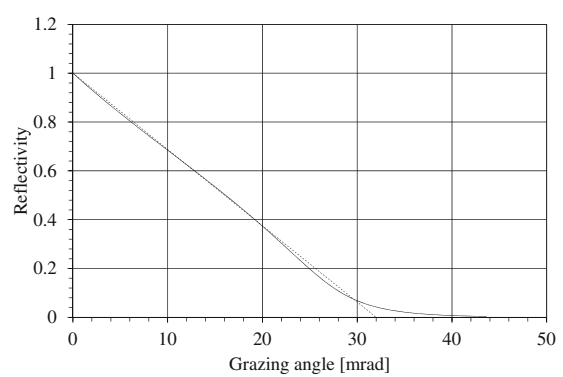

(b) Iridium at $3 \mathrm{keV}$, microrougness $1 \mathrm{~nm}$

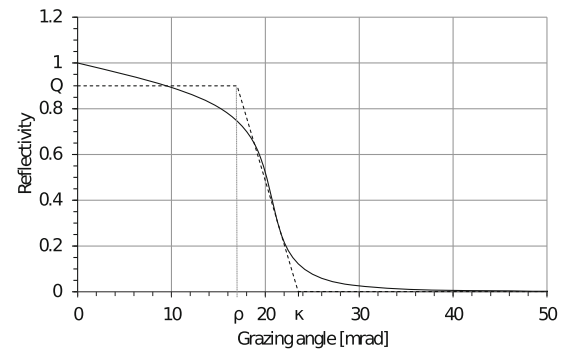

(c) titanium at $2 \mathrm{keV}$

Fig. 6 Examples of relevant approximations (dashed line) of reflectivity data (solid line) 
Graph of $K$ vs. $\zeta$ for mentioned example is shown in Fig. 7. The final equation for the effective collecting length equals

$$
L= \begin{cases}2 r \frac{a}{a+t} Q\left(-\frac{\zeta^{2}}{\kappa}+\zeta\right) & \forall 0 \leq \zeta \leq \frac{\kappa}{2} \\ 2 r \frac{a}{a+t} Q\left(\frac{\zeta^{2}}{3 \kappa}-\zeta-\frac{\kappa^{2}}{6 \zeta}+\kappa\right) & \forall \frac{\kappa}{2} \leq \zeta \leq \kappa \\ 2 r \frac{a}{a+t} Q\left(\frac{\kappa^{2}}{6 \zeta}\right) & \forall \kappa \leq \zeta\end{cases}
$$

The function (19) is maximised and therefore the system has the maximal effective collecting length if

$$
\zeta_{\text {optimal }}=\frac{\kappa}{2}
$$

This result is presented also in paper [4], where a similar model of reflectivity is supposed for a microscope configuration.

\section{Smoothed step model}

Other model of wide usage is

$$
\mathcal{R}(\theta)= \begin{cases}Q & \forall 0 \leq \theta \leq \rho \\ Q \frac{\kappa-\theta}{\kappa-\rho} & \forall \rho \leq \theta \leq \kappa \\ 0 & \forall \theta \geq \kappa\end{cases}
$$

Here, $\theta$ is the grazing angle and $0<\rho<\kappa$ and $0<Q \leq 1$ are constants.

The model represents the typical shape of a reflectivity function. Example of the approximation is shown in Fig. 6c for titanium and unpolarized X-rays of energy

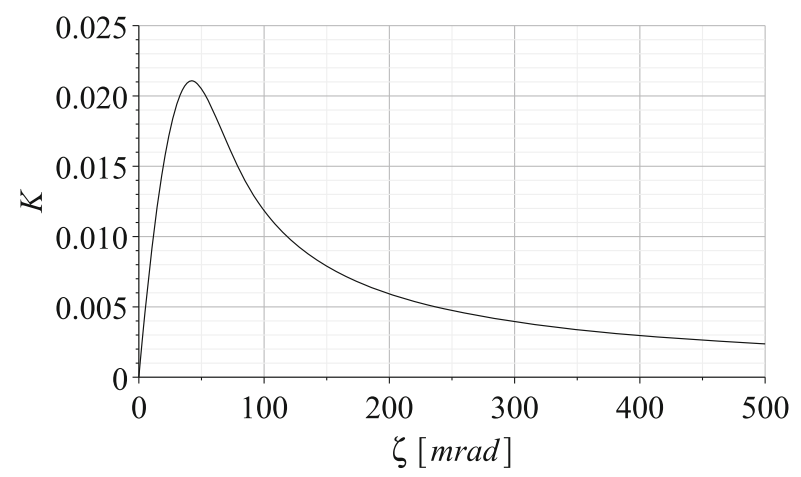

Fig. 7 Example of function $K$ vs. effective angle $\zeta$ for linear model 
$2 \mathrm{keV}$. Here, $Q=0.9, \rho=17 \mathrm{mrad}$ and $\kappa=23.5 \mathrm{mrad}$. Reflectivity data were acquired from [9].

After the substitution $\rho=\kappa \sigma$, where $0<\sigma<1$ and after modifications, the function $K$ equals

$$
K(\zeta)=\left\{\begin{array}{l}
Q J_{1}(\zeta) \forall 0 \leq \zeta \leq \sigma \kappa / 2 \\
Q J_{2}(\zeta) \forall \sigma \kappa / 2 \leq \zeta \leq \sigma \kappa \\
Q J_{4}(\zeta) \forall \sigma \kappa \leq \zeta \leq \kappa / 2 \\
Q J_{5}(\zeta) \forall \kappa / 2 \leq \zeta \leq \kappa \\
Q J_{6}(\zeta) \forall \zeta \geq \kappa \\
Q J_{1}(\zeta) \forall 0 \leq \zeta \leq \sigma \kappa / 2 \\
Q J_{2}(\zeta) \forall \sigma \kappa / 2 \leq \zeta \leq \kappa / 2 \\
Q J_{3}(\zeta) \forall \kappa / 2 \leq \zeta \leq \sigma \kappa \\
Q J_{5}(\zeta) \forall \sigma \kappa \leq \zeta \leq \kappa \\
Q J_{6}(\zeta) \forall \zeta \geq \kappa
\end{array}\right\} \text { if } \quad \sigma \leq 1 / 2
$$

Where

$$
\begin{aligned}
& J_{1}=\zeta \\
& J_{2}=\frac{4 \zeta^{2}}{3 \kappa(\sigma-1)}-\frac{2 \zeta}{\sigma-1}-\zeta-\frac{\sigma^{3} \kappa^{2}}{6(\sigma-1) \zeta}+\frac{\sigma^{2} \kappa}{\sigma-1} \\
& J_{3}=-\zeta-\frac{\kappa^{2}\left(\sigma^{3}-1\right)}{6(\sigma-1) \zeta}+\frac{\kappa\left(\sigma^{2}-1\right)}{\sigma-1} \\
& J_{4}=\frac{\zeta^{2}}{\kappa(\sigma-1)}-\frac{\zeta}{\sigma-1}+\frac{\sigma^{3} \kappa^{2}}{6(\sigma-1) \zeta} \\
& J_{5}=-\frac{\zeta^{2}}{3 \kappa(\sigma-1)}+\frac{\zeta}{\sigma-1}+\frac{\kappa^{2}\left(\sigma^{3}+1\right)}{6(\sigma-1) \zeta}-\frac{\kappa}{\sigma-1} \\
& J_{6}=\frac{\kappa^{2}\left(\sigma^{3}-1\right)}{6(\sigma-1) \zeta} .
\end{aligned}
$$

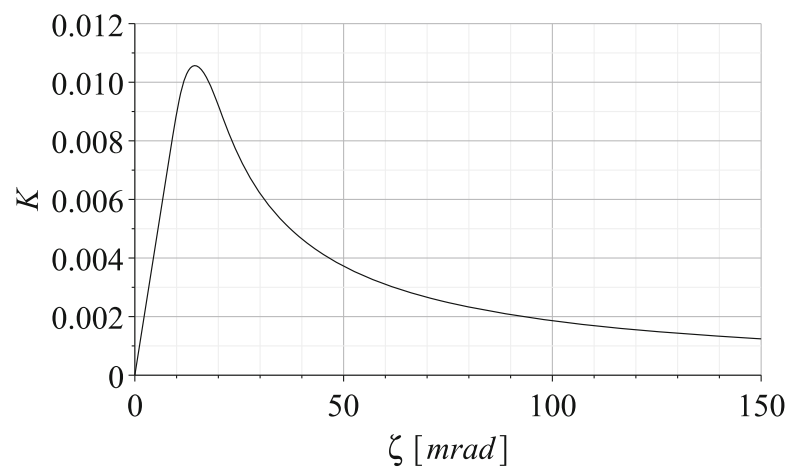

Fig. 8 Example of function $K$ vs. effective angle $\zeta$ for smoothed step model 
Graph of $K$ vs. $\zeta$ for mentioned example is shown in Fig. 8.

Searching for maximum of function (23) is described in the Appendix. We conclude that the function $K(\zeta)$ and consequently the effective collecting length is maximised when

$$
\zeta_{\text {optimal }}= \begin{cases}\left\{\cos \left[\frac{1}{3} \arccos \left(\sigma^{3}\right)+\frac{\pi}{3}\right]+\frac{1}{2}\right\} \kappa & \text { if } \sigma \leq \sigma_{c} \\ \sqrt{6\left(\sigma^{2}+\sigma+1\right)} \frac{\kappa}{6} & \text { if } \sigma \geq \sigma_{c}\end{cases}
$$

where $\sigma_{c}=\frac{1+\sqrt{21}}{10} \doteq 0.558$. As in previous cases, the effective collecting length equals $L(r, a, t, \zeta)=2 r \frac{a}{a+t} K(\zeta)$.

\section{Comparison to ray-tracing simulation}

\subsection{Example 1}

Smoothed step model shown in Fig. 6c with parameters mentioned in the previous section was chosen to be compared with results of numerical simulations based on simplified ray-tracing algorithm (SRTA) [20]. This simulation method is exact. The only approximation was usage of tabulated reflectivity data acquired from [9].

Two different configurations were chosen: a) $r=524 \mathrm{~mm}, a=0.3 \mathrm{~mm}, t=$ $0.1 \mathrm{~mm}, N=100$; b) $r=880 \mathrm{~mm}, a=1.7 \mathrm{~mm}, t=0.3 \mathrm{~mm}, N=120$. Calculations were performed for different values of $\zeta$ with step $1 \mathrm{mrad}$. Results are shown on Fig. 9. Optimal value of $\zeta$ calculated by (30) is $14.4 \mathrm{mrad}$. Analytical calculations show maximum at $14 \mathrm{mrad}$ for both configurations.

\subsection{Example 2}

As an another example, Angel micropore lobster eye of following parameters was chosen: radius $r=800 \mathrm{~mm}$, pore width $a=26 \mu \mathrm{m}$, wall width $t=4 \mu \mathrm{m}$. Calculations were performed for different values of $\zeta$ with step $1 \mathrm{mrad}$. Number of pores in each dimension was set individually for each value of $\zeta$. Minimal number of pores was calculated by (6), the value was rounded up and 10 pores were added. Because the system is two-dimensional, its effective collecting area $S$ equals to square of (11), i.e.

$$
S(r, a, t, \zeta)=\left[2 r \frac{a}{a+t} \frac{\widetilde{\mathcal{R}}(2 \zeta)-2 \widetilde{\mathcal{R}}(\zeta)+\widetilde{R}(0)}{\zeta}\right]^{2}
$$

Pore walls are supposed to be covered by iridium of microrougness $1 \mathrm{~nm}$ and photon energy of $3 \mathrm{keV}$ is assumed. The linear approximation presented in Section 5 was used. Results of analytical model compared to results of simplified ray-tracing algorithm (SRTA) [20] are shown in Fig. 10. Optimal value of $\zeta$ calculated by (21) equals 16 mrad. The analytical as well as the ray-tracing calculations show maxima at this value. 


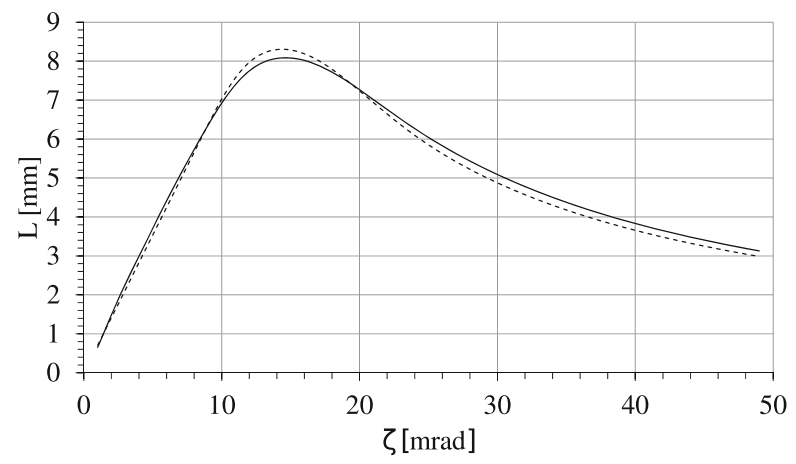

(a) configuration a)

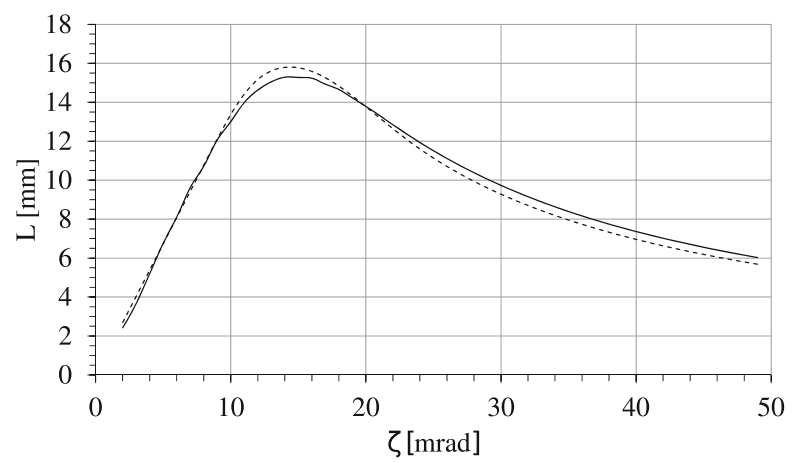

(b) configuration b)

Fig. 9 Comparison of results of ray-tracing simulations (solid line) and presented analytical method (dashed line) for the single Schmidt stack. Graphs of effective collecting length $L$ vs. effective angle $\zeta$

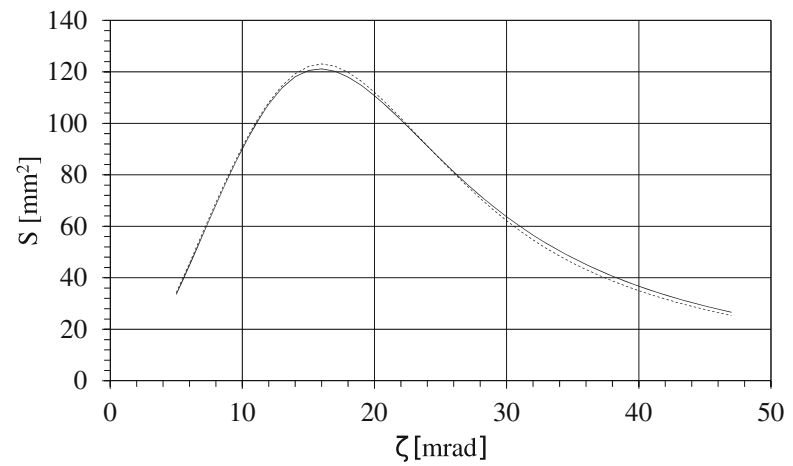

Fig. 10 Comparison of results of ray-tracing simulations (solid line) and presented analytical method (dashed line) for the Angel micropore system. Graph of effective collecting area $S$ vs. effective angle $\zeta$ 


\section{Conclusions}

General (11) for calculating the effective collecting length of one-dimensional lobster systems or the effective collecting area of two-dimensional lobster systems is presented. The equation is simple and compact and gives an understanding of the dependence of effective collecting area (length) versus lobster eye geometrical parameters. It has been proven that optimal ratio between mirrors depth and distance (effective angle) does not depend on other geometrical parameters and it is determined only by reflectivity function, i.e. by mirrors (or their coating) material and photon energy. The entire system is supposed in telescope configuration, i.e. an X-ray source is at infinity.

The general (11) was applied to three reflectivity models that are applicable to wide range of materials. Formulae for effective collecting length (area) for these models were derived: Eq. 16 for single step model, Eq. 20 for linear model and (23)(29) for smoothed step model. Equations for optimal value of $\zeta$ were derived: Eq. 17 for single step model, Eq. 21 for linear model and (30) for smoothed step model. The presented equations can be used for approximate but fast estimation of performance and for finding the initial point for consequent optimization by ray-tracing simulations.

Numerical results are compared to results of ray-tracing simulations and they show excellent accordance.

Acknowledgments For the financial support, we thank to AHEAD (Integrated Activities in the High Energy Astrophysics Domain) project funded by the European Union as Research and Innovation Action under Grant No: 654215. We would like to thank the Grant Agency of the Czech Republic for the financial support by grant number 13-33324S.

Open Access This article is distributed under the terms of the Creative Commons Attribution 4.0 International License (http://creativecommons.org/licenses/by/4.0/), which permits unrestricted use, distribution, and reproduction in any medium, provided you give appropriate credit to the original author(s) and the source, provide a link to the Creative Commons license, and indicate if changes were made.

\section{Appendix : Searching for optimal value of $\zeta$ in smoothed step model}

Analysis of first derivatives showed that $K^{\prime}(\zeta)=Q$ for all $\zeta \in[0, \sigma \kappa / 2]$ and $K^{\prime}\left(\zeta_{C}\right)<0$, where $\zeta_{c}:=\frac{\sqrt[3]{4 \sigma^{3}+4}}{2} \kappa$. The apostrophes denote derivatives by $\zeta$. Brackets [] denote a closed interval while ( ) are used for an open interval.

Analysis of second derivatives showed that $K^{\prime \prime}(\zeta)=0$ for all $\zeta \in[0, \sigma \kappa / 2]$ and $K^{\prime \prime}\left(\zeta_{C}\right)=0$. In addition, $K^{\prime \prime}(\zeta)<0$ for all $\zeta \in\left(\sigma \kappa / 2, \zeta_{c}\right)$ and $K^{\prime \prime}(\zeta)>0$ for all $\zeta \in\left(\zeta_{c},+\infty\right)$.

Function $K$ has continuous second derivatives and there does not exist a point $\zeta$, where $K^{\prime}(\zeta)=K^{\prime \prime}(\zeta)=0$. Therefore, the maximum of function $K$ can lay only at border point 0 (that is not a maximum evidently) or at points, where $K^{\prime}=0$ and $K^{\prime \prime}<0$. Because the interval $\left(\sigma \kappa / 2, \zeta_{C}\right)$ represents the only set of points, where 
$K^{\prime \prime}<0$, the function $K$ has only one maximum and the maximum lies in interval $\left(\sigma \kappa / 2, \zeta_{c}\right)$.

A) Let us analyse

$$
J_{3}^{\prime}=\frac{\kappa^{2}\left(\sigma^{3}-1\right)}{6 \zeta^{2}(\sigma-1)}-1 .
$$

It equals zero at points $\pm \sqrt{6\left(\sigma^{2}+\sigma+1\right)} \kappa / 6$. The negative point does not represent a searched solution. The function $J_{3}$ is to be applied only on interval $[\kappa / 2, \sigma \kappa]$ and if $\sigma \geq 1 / 2$. Therefore, it is necessary to check if $\zeta_{\text {optimal }}=$ $\sqrt{6\left(\sigma^{2}+\sigma+1\right)} \kappa / 6$ belongs to this interval. It does, if $\sigma \geq \sigma_{c}$, where $\sigma_{c}=$ $(1+\sqrt{21}) / 10$.

B) Let us analyse

$$
J_{5}^{\prime}=\frac{\kappa^{2}\left(\sigma^{3}+1\right)}{6 \zeta^{2}(1-\sigma)}+\frac{2 \zeta}{3 \kappa(1-\sigma)}-\frac{1}{1-\sigma} .
$$

Searching for points, where $J_{5}^{\prime}=0$ leads to an equation

$$
\zeta^{3}-\frac{3 \kappa}{2} \zeta^{2}+\frac{\kappa^{3}\left(1+\sigma^{3}\right)}{4}=0 .
$$

The cubic (34) was solved by method given in [16]. The determinant of (34) equals $\Delta=\kappa^{6}\left(\sigma^{6}-1\right) / 64<0$. Therefore, Eq. 34 has three different real roots. After substitutions $\zeta=t+\kappa / 2, t=y+\kappa^{2} /(4 y)$, the following equation for unknown $y \in \mathbb{C}$ is obtained

$$
y^{6}+\frac{\sigma^{3} \kappa^{3}}{4} y^{3}+\frac{\kappa^{6}}{64}=0 .
$$

This is a quadratic equation for $y^{3}$ having six solutions

$$
y_{n}=\frac{\kappa}{2} \sqrt[3]{-\sigma^{3} \pm \sqrt{\sigma^{6}-1}} \exp (\text { ( } 2 n \pi / 3) \quad ; n \in\{0,1,2\}
$$

After backward substitutions, three possible solutions for $\zeta \in \mathbb{R}$ are obtained. It does not make a difference if + or - before the square root in (36) is chosen.

$$
\zeta_{n}=\left\{\cos \left[\frac{1}{3} \arccos \left(-\sigma^{3}\right)+\frac{2 n \pi}{3}\right]+\frac{1}{2}\right\} \kappa \quad ; n \in\{0,1,2\} .
$$

The function $J_{5}$ is being applied only if $\zeta \in[\kappa / 2, \kappa]$ and $\sigma \leq 1 / 2$ or $\zeta \in[\sigma \kappa, \kappa]$ and $\sigma \geq 1 / 2$. Moreover, the root must belong to interval $\left(\sigma \kappa / 2, \zeta_{c}\right)$. These conditions are possible to fulfil only if $\sigma \leq \sigma_{c}$ and the desired root is $\zeta_{2}$ only. It equals

$$
\zeta_{\text {optimal }}=\left\{\cos \left[\frac{1}{3} \arccos \left(\sigma^{3}\right)+\frac{\pi}{3}\right]+\frac{1}{2}\right\} \kappa
$$

For each $0<\sigma<1$, a maximum of function $K(\zeta)$ has been found. Because it was proved that the function $K(\zeta)$ has only one maximum, this is the global maximum.

Publisher's note Springer Nature remains neutral with regard to jurisdictional claims in published maps and institutional affiliations. 


\section{References}

1. Owens, A., Bavdaz, M., Beijersbergen, M.W., Brunton, A.N., et al.: Hermes: an imaging x-ray fluorescence spectrometer for the bepicolombo mission to mercury, pp. 4506-10 (2001)

2. Angel, J.R.P.: Lobster eyes as X-ray telescopes. ApJ 233, 364-373 (1979)

3. Baca, T., Platkevic, M., Jakubek, J., Inneman, A., Stehlikova, V., Urban, M., Nentvich, O., Blazek, M., McEntaffer, R., Daniel, V.: Miniaturized X-ray telescope for VZLUSAT-1 nanosatellite with Timepix detector. J. Instrum. 11, C10007 (2016)

4. Chapman, H.N., Nugent, K.A., Wilkins, S.W.: X-ray focusing using square channel-capillary arrays. Rev. Sci. Instrum. 62(6), 1542-1561 (1991)

5. Collier, M.R., Porter, F.S., Sibeck, D.G., Carter, J.A., Chiao, M.P., Chornay, D.J., Cravens, T.E., Galeazzi, M., Keller, J.W., Koutroumpa, D., Kujawski, J., Kuntz, K., Read, A.M., Robertson, I.P., Sembay, S., Snowden, S.L., Thomas, N., Uprety, Y., Walsh, B.M.: Invited article: first flight in space of a wide-field-of-view soft X-ray imager using lobster-eye optics: instrument description and initial flight results. Rev. Sci. Instrum. 86(7), 071301 (2015)

6. Fraser, G.W., Brunton, A.N., Bannister, N.P., Pearson, J.F., Ward, M., Stevenson, T.J., Watson, D.J., Warwick, B., Whitehead, S., O’Brian, P., White, N., Jahoda, K., Black, K., Hunter, S.D., DeinesJones, P., Priedhorsky, W.C., Brumby, S.P., Borozdin, K.N., Vestrand, T., Fabian, A.C., Nugent, K.A., Peele, A.G., Irving, T.H., Price, S., Eckersley, S., Renouf, I., Smith, M., Parmar, A.N., McHardy, I.M., Uttley, P., Lawrence, A.: LOBSTER-ISS: an imaging X-ray all-sky monitor for the international space station. In: Flanagan, K.A., Siegmund, O.H.W. (eds.) Proceedings of the SPIE X-Ray and Gamma-Ray Instrumentation for Astronomy XII, vol. 4497, pp. 115-126 (2002)

7. Gorenstein, P.: Large-angle observatory with energy resolution for synoptic X-ray studies (LOBSTER-SXS). In: Proceedings of the Society of Photo-Optical Instrumentation Engineers (SPIE) Conference Series, vol. 8147, p. 814710 (2011)

8. Fraser, G.W., Carpenter, J.D., Rothery, D.A., et al.: The mercury imaging X-ray spectrometer (mixs) on bepicolombo. Planet. Space Sci. 58(1), 79-95 (2010)

9. Henke: http://henke.lbl.gov/optical_constants/ (2018)

10. Inneman, A.: Technologické aspekty vývoje a výroby optických prvků pro rentgenové záření. Ph.D. thesis, Czech Technical University in Prague Faculty of Mechanical Engineering (2001)

11. Petre, R., Camp, J., Barthelmy, S., Gehrels, N., Racusin, J., Marshall, F., Ptak, A.: ISS-Lobster: a low-cost wide-field X-ray transient detector on the ISS. In: APS Meeting Abstracts (2015)

12. Schmidt, W.K.H.: A proposed X-ray focusing device with wide field of view for use in X-ray astronomy. Nucl. Inst. Methods 127, 285-292 (1975)

13. Spencer, G.H., Murty, M.V.R.K.: General ray-tracing procedure. J. Opt. Soc. Am. (1917-1983) 52, 672 (1962)

14. Spiga, D.: Optics for X-ray telescopes: analytical treatment of the off-axis effective area of mirrors in optical modules. A\&A 529, A18 (2011)

15. Spiga, D., Cotroneo, V., Basso, S., Conconi, P.: Analytical computation of the off-axis effective area of grazing incidence X-ray mirrors. A\&A 505, 373-384 (2009)

16. Stedall, J.A.: The greate invention of algebra: Thomas harriot's treatise on equations. Oxford University Press, Oxford (2003)

17. Su, L., Li, W., Wu, M., Su, Y., Guo, C., Ruan, N., Yang, B., Yan, F.: Modeling the focusing efficiency of lobster-eye optics for image shifting depending on the soft x-ray wavelength. Appl. Opt. 56(22), 6267-6271 (2017)

18. Tichý, V., Burrows, D.N., Prieskorn, Z., Hudec, R.: Optics for nano-satellite X-ray monitor. Balt. Astron. 24, 243-250 (2015)

19. Tichý, V., Hudec, R., Barbera, M.: Analytical description of lobster eye and similar multi-foil optics. In: EUV and X-Ray Optics: Synergy between Laboratory and Space IV, Proc. SPIE, vol. 9510, p. 95100C (2015)

20. Tichý, V., Hudec, R., Němcová, Š.: Effective algorithm for ray-tracing simulations of lobster eye and similar reflective optical systems. Exp. Astron. 41, 377-392 (2016)

21. Šaroun, J., Kulda, J.: MC ray-tracing optimization of lobster-eye focusing devices with RESTRAX. Phys. B Condens. Matter 385, 1250-1252 (2006) 